\title{
Using positron emission tomography (PET) to determine liquid content in overflowing foam
}

K. Cole ${ }^{1 *}$, P.R. Brito-Parada ${ }^{1 *}$, A. Morrison ${ }^{1}$, I. Govender ${ }^{2}$, A. Buffler ${ }^{2}$, K. Hadler $^{1}$, J.J. Cilliers ${ }^{1}$

${ }^{1}$ Rio Tinto Centre for Advanced Mineral Recovery, Department of Earth Science and Engineering, Imperial College London, UK.

${ }^{2}$ Department of Physics, University of Cape Town, South Africa.

*Corresponding author: katie.cole@uct.ac.za. Present address: ${ }^{2}$

\section{Abstract:}

In froth flotation, liquid recovery is strongly linked to separation performance. In addition to determining its structure, the distribution of liquid within a froth affects the recovery of entrained material to the concentrate. Despite its importance, measurements of the liquid content of froths are rare. Most existing measurements are intrusive, only provide low spatial resolution, or rely on small samples of foam. This work demonstrates for the first time the use of positron emission tomography (PET) to measure the distribution of liquid in a 2D overflowing foam. Results show a liquid fraction of 0.36 at the base of the foam column decreasing to a liquid fraction of 0.03 at the foam surface. This shows the potential to use PET studies to measure 3D foam structures and to validate simulations of flotation froths.

\section{Graphical Abstract:}
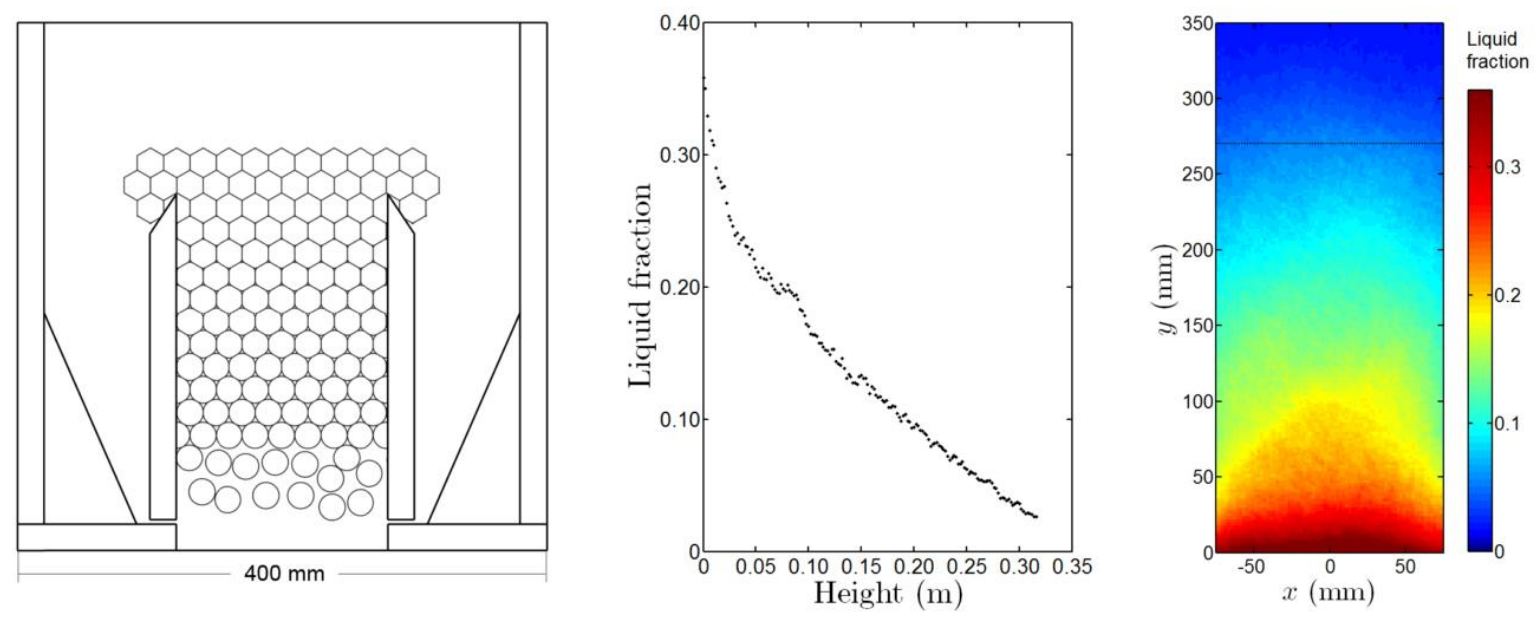

Keywords: PET; foam; froth; liquid fraction

\section{Highlights}

- Liquid content measurements in foams and froths are limited by their opaque nature

- Positron emission tomography was used to measure the dispersion of a liquid tracer

- The density of PET lines of response was used to find the liquid content of 2D foam

- The liquid content at the interface was 0.36 decreasing to 0.03 at the surface

- This technique can be applied to liquid content in 3D foams and flotation froths 


\section{Introduction}

In flotation, recovery of non-selectively entrained gangue to the concentrate is directly proportional to the recovery of water (Engelbrecht and Woodburn, 1975; Neethling et al., 2000). The liquid dispersion within the Plateau border network of the froth has been shown to have a significant impact on the amount and purity of the concentrate (Lee et al., 2003). This makes liquid content an important variable in numerical modelling of flotation froth behaviour, such as by Brito-Parada et al. (2012), who implemented a model for foam drainage to solve transient liquid motion in 3D foams. However, experimental validation of such simulations has proven challenging due to the opaque and fragile nature of froths and foams (Malysa, 1998).

Liquid content in a two-phase foam has previously been measured with bubble pressure gauges or manometers, where the maximum pressure to form a bubble is correlated with the liquid content (Cutting et al., 1981; Ireland \& Jameson, 2007). However, these measurements are intrusive and may alter the froth structure. Measurements of foam electrical conductivity and conductance do not have this limitation and can be applied outside of the vessel, as the ability of a foam to conduct electricity varies with the distribution and concentration of liquid (Lemlich, 1978; Karapantsios \& Papara, 2008). Electrical resistance tomography (ERT) can also be used non-intrusively to find areas of different liquid concentration within a foam (Wang \& Cilliers, 1999; Cilliers et al., 2001; Xie et al., 2004). However, ERT has only produced low resolution images of liquid content in foams; of the order of several centimetres.

Hartman \& Barber (1974) measured the liquid hold up in a foam column up with radioactive tracers. The foaming system was dosed with ${ }^{22} \mathrm{NaCl}$, which emits gamma radiation at $0.51 \mathrm{MeV}$ and 1.08 $\mathrm{MeV}$. The $0.51 \mathrm{MeV}$ gamma rays were detected by a scintillation counter at a specific height in the foam. Counts from a calibration solution were used to correct for background counts from the liquid solution at the base of the column. The liquid hold up values were used to find the film thickness at a range of surfactant concentrations and gas velocities. Recently, X-ray tomography and radioscopy have been used to measure significant changes in liquid content from changes in density with time, whilst additionally revealing the internal structure of foams on the micro-scale. Davies et al. (2013) used X-ray micro-tomography to image a small sample of foam at very high spatial resolution, and Solórzano et al. (2013) used X-ray radioscopy in two modes to measure the liquid content and bubble size of beer foams. So far, these X-ray techniques rely on small active areas and, as such, have only been applied to small experimental systems.

Positron emission tomography (PET) is another non-intrusive, penetrative measurement technique that has been used to successfully image liquid flow in engineering systems (Hawkesworth et al., 1991; Parker \& McNeil, 1996). A liquid tracer is made by labelling the liquid phase of the system with a positron emitting radionuclide which does not adversely interact with the chemical and physical behaviour of the system. This can be used in two ways: by injecting a pulse of tracer into the system to follow its dispersion and flow with time, or by allowing the tracer to completely disperse throughout the system to measure the distribution or density of tracer at steady state.

The decay of the radionuclide in the tracer produces positrons, which travel a short distance before annihilation with local electrons. This event produces pairs of almost back-to-back gamma rays with energy $511 \mathrm{keV}$. If both gamma rays in each pair are detected in coincidence by detector blocks in the PET camera, a virtual line of response can be formed between the two detector blocks. As the gamma rays originated at some point along this line, multiple lines of response can be back- 
projected (Chesler, 1973) to form 2D sinograms (Townsend et al., 1989) and reconstructed into a 3D image of the distribution of liquid tracer.

A new research centre for positron imaging, called PEPT Cape Town, was opened at the University of Cape Town in 2009 (Buffler et al., 2010). It houses an ECAD "EXACT3D" HR++ positron emission tomography camera, which has an extended cylindrical geometry. The camera is highly sensitive as it contains a large number of detector elements and can achieve spatial resolutions of a few millimetres. The field of view of this camera is a cylinder with diameter $600 \mathrm{~mm}$ and axial length 200 $\mathrm{mm}$, which allows large laboratory scale systems to be imaged with PET.

This paper presents the first application of PET and liquid tracers to overflowing foams to determine the liquid fraction throughout a 2D foam.

\section{Experimental Methods}

PET experiments were performed at PEPT Cape Town using an overflowing 2D foam column developed by Cole (2011) and Cole et al. (2012b). This consists of a vertical monolayer of foam between parallel plates of Perspex, separated by an air gap of $5 \mathrm{~mm}$. Air was introduced between the plates by a network of capillary tubes, to provide a uniform air distribution to the base of the column. The bubbles produced at the capillary tubes formed randomly and loosely packed elliptical cylinders between the parallel plates, with approximate values for the semi-major axis of $2.7 \mathrm{~mm}$ and semi-minor axis $1.8 \mathrm{~mm}$. These bubbles were 2D according to the relation for critical plate separation suggested by Cox et al. (2002). The average cross-section of the bubbles became circular within the first $5 \mathrm{~cm}$ of the height of the foam, and the bubbles were loosely packed in a hexagonal configuration. At the top of the foam, the bubbles formed polygonal prisms with an increase in bubble size due to coalescence. A schematic of the column is given in Figure 1 (left), showing an internal section for the rising foam, two sloping weirs for overflow, and areas for the liquid to be recycled.

Air recovery (the fraction of air entering the cell that overflows in unburst bubbles) was estimated to be $60 \%$ from image analysis, and the average overflowing foam height above the lip was $100 \mathrm{~mm}$. The foam was more stable than has been produced previously, as in Cole (2011), with less bubble coalescence at the base of the column and a higher air recovery. One reason for this effect could be the gallium ions acting at the bubble interfaces to increase electric double layer repulsive forces, which causes the films to drain more slowly, resulting in less coalescence (Craig, 2004). Online measurements of the foam behaviour were not possible due to health and safety restrictions to minimise worker contact with the radioactive fluid. 

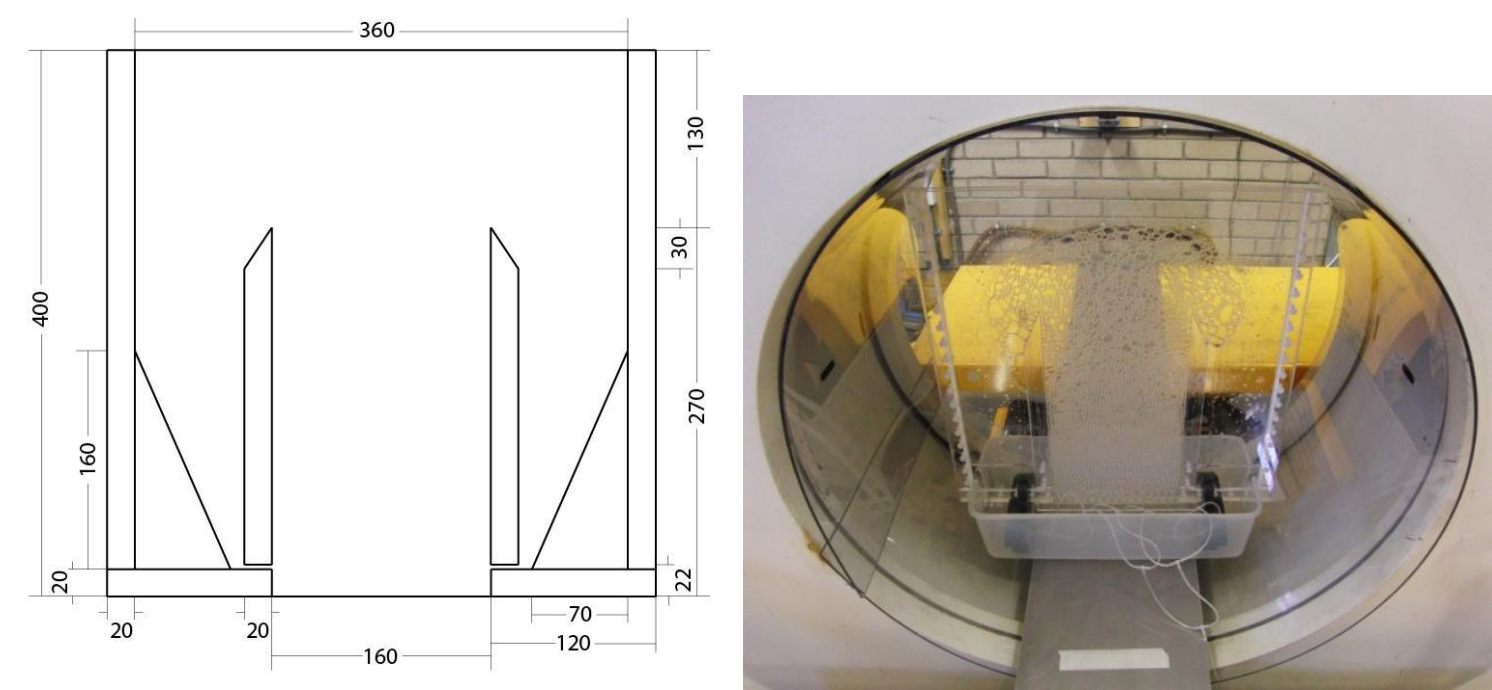

Figure 1: Schematic of the 2D overflowing foam column with measurements in millimetres (left), and photograph of the column inside the positron camera (right).

As shown in Figure 1 (right), the column was mounted in the field of view of the positron camera by a $5 \mathrm{~mm}$ aluminium sheet suspended between two mounts, and experiments were performed under the conditions given in Table 1. A polycarbonate shield was placed around the inside of the camera to protect the camera from stray liquid.

The radionuclide used for PET studies was ${ }^{68} \mathrm{Ga}$, which has a half life of approximately 68 minutes. The ${ }^{68} \mathrm{Ga}$ was eluted from a ${ }^{68} \mathrm{Ge}$ generator with dilute hydrochloric acid as described in Cole et al. (2012a). This small volume of tracer was then diluted with $60 \mathrm{ml}$ of the foaming solution to be syringed into the top of the foam column. The foam was left running for 20 minutes to allow the tracer to be uniformly distributed throughout the column.

Table 1: Experimental conditions and quantities

\begin{tabular}{|ll|}
\multicolumn{1}{c|}{ Condition } & \multicolumn{1}{c|}{ Quantity } \\
\hline Ambient temperature / humidity & $22.1^{\circ} \mathrm{C} / 55 \%$ \\
\hline Frother & $2 \mathrm{~g} / \mathrm{l}$ methyl isbutyl carbinol \\
\hline Viscosity modifier & $0.1 \mathrm{~g} / \mathrm{l}$ xanthan gum \\
\hline Air rate & $1.75 \mathrm{lpm}$ \\
\hline Initial activity & $1.1 \mathrm{mCi}(40.7 \mathrm{MBq})^{68} \mathrm{Ga}$ \\
\hline Tracer elution & $160 \mu \mathrm{l}$ with $0.6 \mathrm{M} \mathrm{HCl}$ \\
\hline Final solution volume & $60 \mathrm{ml}$ \\
\hline
\end{tabular}

The raw listmode data was recorded by the ECAD "EXACT3D" HR++ positron emission tomography camera (Model: CTI/Siemens 966) over 60 seconds. A simple back-projection method developed by Bickell (2012) and Bickell et al. (2012) was applied to the list mode data from the positron camera. This counted the number of lines of response that passed through each $2 \mathrm{~mm}$ cubic voxel of a Cartesian mesh over the field of view. This line density is proportional to the density or concentration of the tracer in each voxel, and thus the amount of liquid present. 


\section{Results and discussion}

A vertical 2D slice of the 3D line density data from PET over a grid of voxel size $2 \mathrm{~mm}$ is plotted in Figure 2. The line density at the base of the column was high, which corresponds with a bubbly liquid layer as shown in Figure 1 (right). The line density then decreased with the formation of foam upwards through the column. Non-zero values of line density are shown in areas of the column where liquid was not present. This noise is a result of counting voxels through which lines of response pass, but do not necessarily originate. A mean average of these values was selected as zero liquid content. The highest line density was measured in the recycle, and was chosen as the value of $100 \%$ liquid content. The line density values corresponding to 0 and $100 \%$ liquid content were used to calculate the liquid content as a fraction elsewhere in the foam column.

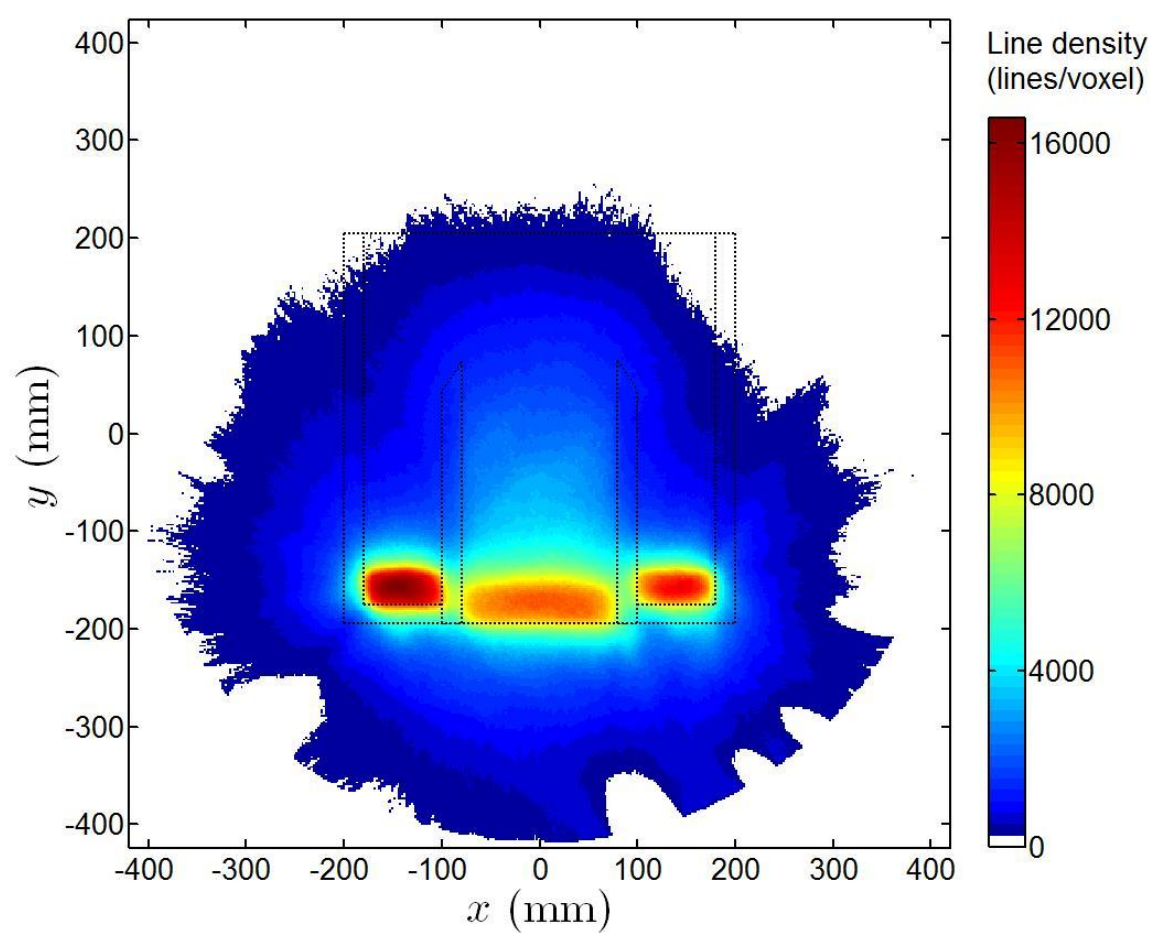

Figure 2: Line density in the overflowing foam column.

The relationship between liquid fraction with height in the centre of the foam column is shown in Figure 3, starting at the height of the interface. The liquid fraction dropped off rapidly at the base of the foam, and more gradually towards the lip where the foam overflowed. The liquid fraction at the liquid-foam interface was 0.36 , decreasing to 0.03 by the top of the foam. The packing structure of 2D foam can be compared to the void fraction of packed structures formed of elliptical and circular discs, at the base and mid-height of the foam respectively. According to geometry, the voidage of both mono-disperse elliptical and circular discs in close packed hexagonal structures is approximately $10 \%$. The liquid fraction of the foam tended to this voidage at a height of $15 \mathrm{~cm}$, indicating the foam was closely packed at this height. However at the base of the column, the liquid fraction was higher which indicates the foam was wetter with a looser packing structure. The liquid fraction values of a foam trapped between plates will tend to be larger than the voidages of hard discs, due to liquid trapped between the bubbles and the walls of the column, changes in bubble size and shape, and elastic deformations to the bubbles during packing at the point of foam formation. 


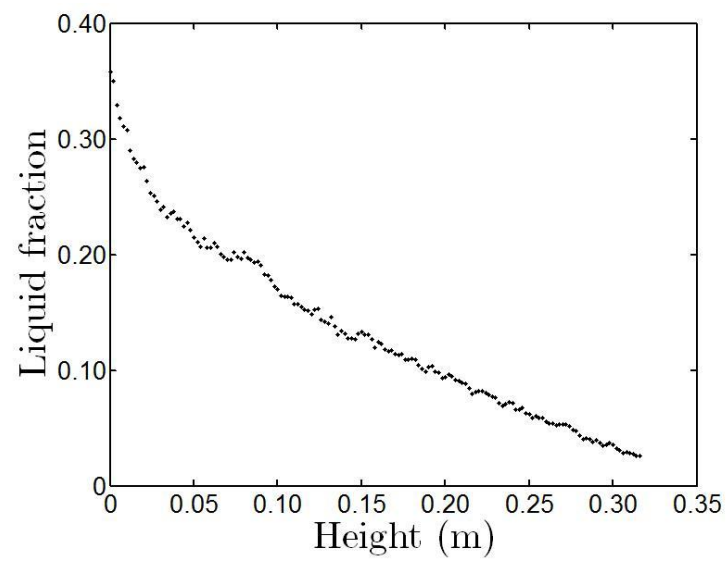

Figure 3: Liquid fraction with height in the centre of the column measured with PET.

The liquid fraction in the foam was calculated above the interface as shown in Figure 4. This calculation assumed that the line density from PET was directly proportional to the liquid content and the tracer was completely dispersed throughout the foam at steady state. These results show the measured liquid fraction was higher in the centre compared to near the column wall. This suggests there were strong wall effects resulting in a drier foam.

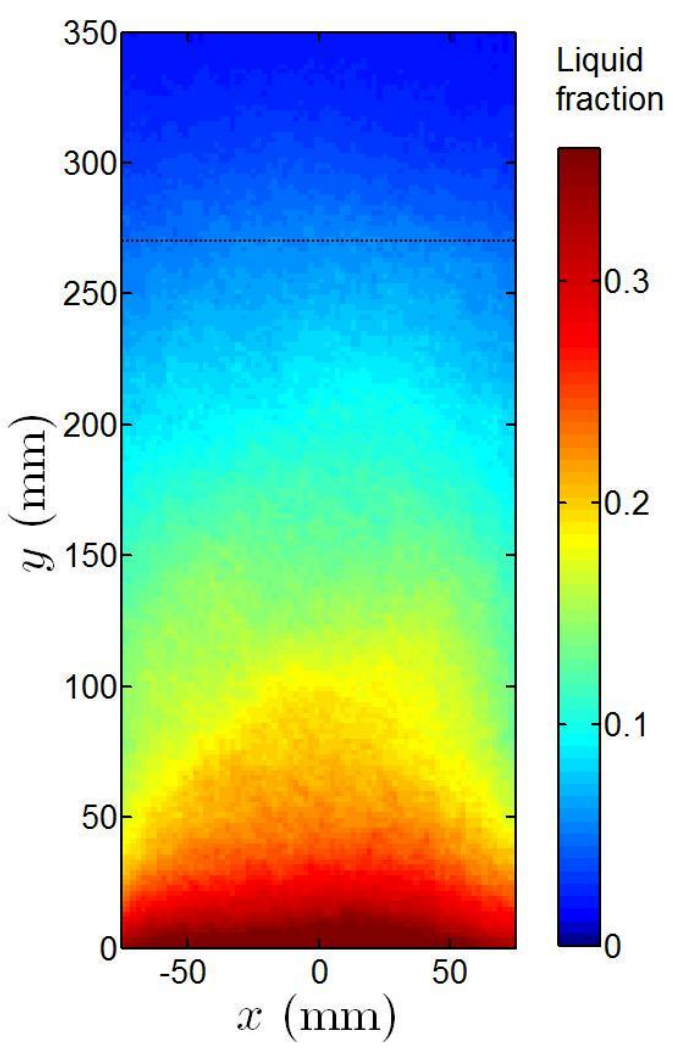

Figure 4: Liquid fraction in the 2D overflowing foam column from the interface measured with PET (-indicates the lip level). 
These experimental results highlight the potential of using PET to measure liquid content in flowing foams, and can be compared to image analysis techniques to explain changes in bubble size distribution and air recovery. In future, the liquid content measurements can be improved by removing background counts from the liquid solution at the base of the column (Hartland \& Barber, 1974), filtering methods such as ramp and low pass filters (Bickell, 2012), and attenuation correction (Saha, 2005).

\section{Conclusion}

The liquid content in an overflowing 2D foam has been determined with positron emission tomography (PET) for the first time. The liquid fraction at the base of the column was found to be 0.36, corresponding to a foam structure composed of loosely packed elliptical cylinders. The liquid fraction at the bursting surface of the foam was 0.03 where the bubbles were packed in polygonal prisms. The liquid fraction decreased as the froth height increased, with a higher fraction in the centre of the column and lower liquid fraction near the walls.

This study demonstrates the potential for PET to be used to investigate liquid content in 2D foams including convective rolls and forced drainage. With image analysis techniques these measurements will help to explain changes in bubble size due to coalescence. They can also be extended to measurements in 3D and three phase systems, to investigate the characteristics of the gas phase and air recovery.

\section{Acknowledgements}

The financial support of Rio Tinto, through the Rio Tinto Centre for Advanced Mineral Recovery, is gratefully acknowledged. The authors would like to thank M. van Heerden and C. Liu from iThemba LABS for their support in fabricating tracers, and M. Bickell at the Medical Imaging Research Center at KULeuven, Belgium, for his guidance in applying the line density algorithm.

\section{References}

Bickell, M. (2012). Investigations into a positron emission imaging algorithm. MSc thesis. University of Cape Town, South Africa.

Bickell, M., Buffler, A., Govender, I., Parker, D.J. (2012) A new line density tracking algorithm for PEPT and its application to multiple tracers. Nuclear Instruments and Methods in Physics Research A, 682, 36-41.

Brito-Parada, P.R., Neethling, S.J., Cilliers, J.J. (2012). The advantages of using mesh adaptivity when modelling the drainage of liquid in froths. Minerals Engineering, 33, 80-86.

Buffler, A., Govender, I., Cilliers, J.J., Parker, D.J., Franzidis, J.-P., Mainza, A., Newman, R.T., Powell, M., Van der Westhuizen, A., (2010). PEPT Cape Town: a new positron emission particle tracking facility at iThemba LABS. In: Proceedings of International Topical Meeting on Nuclear Research Applications and Utilization of Accelerators, 4-8 May 2009, Vienna (IAEA, Vienna, 2010). STI/ PUB/1433, ISBN 978-92-0-150410-4, ISSN 1991-2374.

Chesler, D.A. (1973). Positron tomography and three-dimensional reconstruction technique. In: Freedman, G.S. (eds.) Tomography Imaging in Nuclear Medicine. The Society of Nuclear Medicine, New York, pp. 176-183.

Cilliers, J. J., Xie, W., Neethling, S. J., Randall, E. W. \& Wilkinson, A. J. (2001) Electrical resistance tomography using a bi-directional current pulse technique. Measurement Science \& Technology, 12, 997-1001. 
Cole, K.E. (2011). Measuring bubble size, coalescence and particle motion in a flowing foam. PhD thesis. Imperial College London, UK.

Cole, K.E., Buffler, A., van der Meulen, N.P., Cilliers, J.J., Franzidis, J-P., Govender, I., Liu, C., van Heerden, M.R. (2012a). Positron Emission Particle Tracking Measurements with 50 micron tracers. Chemical Engineering Science, 75, 235-242.

Cole, K.E., Brito-Parada, P.R., Neethling, S.J., Cilliers, J.J. (2012b) Experimental studies and numerical model validation of overflowing 2D foam to test flotation cell crowder designs. Chemical Engineering Research and Design, 90, 2196-2201.

Cox, S. J., Weaire, D. \& Vaz, M. F. (2002) The transition from two-dimensional to three-dimensional foam structures. European Physical Journal E, 7, 311-315.

Davies, I.T., Cox., S.J., Lambert, J. (2013). Reconstruction of tomographic images of dry aqueous foams. Colloids and Surfaces A: Physicochemical Engineering Aspects, 438, 33-40.

Engelbrecht, J.A., Woodburn, E.T., 1975. The effect of froth height, aeration rate and gas precipitation on flotation. Journal of the South African Institute of Mining and Metallurgy, 10, 125-132.

Saha, G.B. (2005) Basics of PET Imaging. Springer, New York, 39-58.

Hartland, S., Barber, A.D. (1974). A model for a cellular foam. Transactions of the Institution of Chemical Engineers, 52, 43-52.

Hawkeswoth, M.R., Parker, D.J., Fowles, P., Crilly, J.F., Jefferies, N.L., Jonkers, G. (1991). Nonmedical applications of a positron camera. Nuclear Instruments and Methods in Physics Research A, 310, 423-434.

Ireland, P.M., Jameson, G.J. (2007). Liquid transport in a multi-layer froth. Journal of Colloid and Interface Science, 314, 207-213.

Karapantsios, T. D. \& Papara, M. (2008) On the design of electrical conductance probes for foam drainage applications: Assessment of ring electrodes performance and bubble size effects on measurements. Colloids and Surfaces A: Physicochemical and Engineering Aspects, 323, 139-148.

Lee, H.T., Neethling, S.J., Cilliers, J.J. (2005). Particle and liquid dispersion in foams. Colloids and Surfaces A: Physicochemical and Engineering Aspects, 263, 320-329.

Lemlich, R. (1978) A theory for the limiting conductivity of polyhedral foam at low density. Journal of Colloid and Interface Science, 64, 107-110.

Malysa, K. (1998) Chapter 3: Water contents and distribution in flotation froths. In: Laskowski, J., Woodburn, E.T. (eds.) Frothing in Flotation II: Recent Advances in Coal Processing, Volume 2. CRC Press, 81-108.

Neethling, S.J., Cilliers, J.J., Woodburn, E.T. (2000). Prediction of the water distribution in a flowing foam. Chemical Engineering Science, 55, 4021-4028.

Parker, D.J., McNeil, P.A. (1996). Positron emission tomography for process applications. Measurement Science Technology, 7, 287-296.

Solórzano, E., Pardo-Alonso, S., de Saja, J.A., Rodríguez-Pérez, M.A. (2013). Study of aqueous foams evolution by means of X-ray radioscopy. Colloids and Surfaces A: Physicochemical Engineering Aspects, 438, 159-166.

Townsend, D.W., Sprinks, T., Jones, T., Geissbuhler, A., Defrise, M., Gilardi, M.C., Heather, J. (1989). Three dimensional reconstruction of PET data from a multi-ring camera. IEEE Transactions on Nuclear Science, 36 (1), 1056-1065.

Xie, W., Neethling, S. J. \& Cilliers, J. J. (2004) A novel approach for estimating the average bubble size for foams flowing in vertical columns. Chemical Engineering Science, 59, 81-86.

Wang, M. \& Cilliers, J. J. (1999) Detecting non-uniform foam density using electrical resistance tomography. Chemical Engineering Science, 54, 707-712. 